\title{
Strange quark mass dependence of strange quark star properties
}

\author{
Bo-Lin Li ${ }^{1, a}$, Yan Yan ${ }^{2, b}$, Jia-Lun Ping ${ }^{1, c}$ \\ ${ }^{1}$ Department of Physics, Nanjing Normal University, Nanjing 210023, China \\ 2 School of Mathematics and Physics, Changzhou University, Changzhou 213164, Jiangsu, China
}

Received: 3 February 2021 / Accepted: 16 September 2021 / Published online: 20 October 2021

(C) The Author(s) 2021

\begin{abstract}
The effects of strange quark mass on masses, radii, and tidal deformabilities of strange quark stars are studied via the quasiparticle model that includes the nonperturbative features of QCD in low density region. The constraints of $M_{\mathrm{TOV}}>2.0 M_{\odot}$ and $\Lambda_{1.4}<800$ are used to choose the EOSs with different strange quark masses. We find that in low density region the EOSs with larger strange quark mass are stiffer, while in high density region they are softer. A larger strange quark mass gives a smaller mass and radius for a strange quark star, and a larger minimum of radii and tidal deformabilities for $1.4 M_{\odot}$ strange quark stars. A larger strange quark mass also gives a larger lower bound of combined tidal deformability $\tilde{\Lambda}_{L B}$.
\end{abstract}

\section{Introduction}

The three basic properties of a strange quark star are its mass, radius and tidal deformability, which can be calculated once the equation of state (EOS), i.e., the relationship between pressure $p$ and energy density $\epsilon$, of strange quark matter is clarified. In recent years, the observations of the pulsars PSR J0348+0432 $\left(m=2.01 \pm 0.04 m_{\odot}\right)[1]$ and PSR J1614$2230\left(m=1.928 \pm 0.017 m_{\odot}\right)[2]$ give the largest masses of compact stars, which can be used as one of the constraints on our EOSs. On August 17, 2017, the first gravitational wave $(\mathrm{GW})$ signal as well as its electromagnetic counterparts, originating from a binary neutron star system was detected by advanced LIGO and Virgo systems. This event open an new era for the multi-messenger astronomy. The analyses done by the LIGO and Virgo collaborations give the upper bound of the tidal deformability for a $1.4 M_{\odot}$ neutron star, $\Lambda_{1.4}<800$, and the upper bound of the combined tidal deformability for

\footnotetext{
a e-mail: blli@njnu.edu.cn (corresponding author)

be-mail: 2919ywhhxh@163.com

c e-mail: jlping@njnu.edu.cn
}

the two merged stars, $\tilde{\Lambda}<900[3,4]$. These two limits can also be used to constrain the EOS of our model studies.

In the center of a strange quark star, the number density is, $n_{B} \lesssim(4 \sim 7) n_{0}$ where $n_{0} \simeq 0.16$ nucleons per $\mathrm{fm}^{3}$, is the nuclear saturation density. This domain is not that large for the perturbative QCD calculations that is valid for $n_{B} \gtrsim 10-100 n_{0}$ [5]. Hence, many phenomenological models that include some basic characters of QCD are put forward to construct the EOS of strange quark matter in this low density region. The MIT bag model is the widely used one to study the structures of strange quark stars, and it treats quarks as ideal Fermi gases in a bag that has a negative pressure to act as the confinement $[6,7]$. The Nambu-JonaLasinio (NJL) model is also used to describe the properties of quark matter [5,8]. In this paper, a quasiparticle model that treats the microscopic interaction between quarks and gluons as an effective mass modification of each particle, thus incorporates the non-perturbative effects of QCD [9-12], is adopted to construct the equation of state of strange quark matter. We use this model to analyze strange quark mass dependence of strange quark star properties. For different strange quark masses, we calculate the maximum mass and its corresponding radius of strange quark star, the maximum and minimum radius and minimum tidal deformability for a $1.4 M_{\odot}$ strange quark star, and the upper and lower bounds of combined tidal deformability. Since this quasiparticle model is also widely used in studying the properties of quark-gluon plasma (GQP) at finite temperature $(T)$ and chemical potential $(\mu)[13-16,18,21]$, when more analyses of gravitational waves directly detected by LIGO and Virgo collaborations and millisecond pulsars observed by NANOGrav and NICER projects give more precise observations in the future, one could compare the astrophysical and terrestrial calculations within the same model. And constraining the parameters on one side could be used to make predictions for the other side.

This paper is organized as follows: in Sect. 2, we give an introduction to the quasiparticle model and present the formula of the EOS for strange quark matter. While varying the 
bag constant and the coupling strength $\zeta$, using the mass and tidal deformability constraints, the effects of strange quark mass on the mass-radius relations, the tidal deformabilities and the radii for $1.4 M_{\odot}$ strange quark stars, and combined tidal deformabilities $\tilde{\Lambda}$ for GW170817 and GW190425 are presented in detail in Sect. 3. Finally, we give a summary of this work in Sect. 4.

\section{The quasiparticle model}

As we all known, in quantum field theory (QFT), the properties of particles are contained in their full propagators of the theory. When the full quark propagator at zero temperature $T$ and finite chemical potential $\mu$ is specified, the quark number density can be expressed as [17],

$\rho_{f}(\mu)=-N_{c} \int \frac{\mathrm{d}^{4} p}{(2 \pi)^{4}} \operatorname{tr}_{D}\left\{\gamma_{4} S_{f}(p, \mu)\right\}$,

where $S_{f}(p, \mu)$ stands for the full quark propagator, $N_{c}$ is the number of colors which is given by $N_{c}=3$ in this work, and the trace operator " $r_{\mathrm{D}}$ " is taken over all Dirac indices. But, Unfortunately, the exact form of the full quark propagator at zero temperature and finite chemical potential is still unknown from the first principles of QCD. Here, we treat an effective quark propagator that has the same form as the free quark propagator but with a density dependent effective quark mass, as an approximation of the full quark propagator in the dense strange quark matter. And, the effective quark propagator proposed in Ref. [18] is given by,

$S_{f}(p, \mu)=\frac{1}{i \gamma \cdot \tilde{p}+m_{f}(\mu)}$,

where $\tilde{p} \equiv\left(\vec{p}, p_{4}+i \mu\right)$ is the four momenta of the quark at zero temperature and finite chemical potential, and in Euclidean space, the gamma matrices satisfy $\left\{\gamma_{\mu}, \gamma_{\nu}\right\}=$ $2 \delta_{\mu \nu}$. The effective quark mass that depends on chemical potential reads $[9,15]$,

$m_{f}^{2}(\mu)=\left(m_{f 0}+m_{q}(\mu)\right)^{2}+m_{q}^{2}(\mu)$,

with,

$m_{q}^{2}(\mu)=\frac{N_{f} \mu^{2} g^{2}(\mu)}{18 \pi^{2}}$,

where $m_{f 0}$ is the mass of current quark $(u, d$, or $s), N_{f}$ is the number of quark flavors which is given by $N_{f}=3$ in this work. And $g\left(\mu^{2}\right)$ is the effective coupling constant obtained in the two-loop approximation $[16,19-21]$, which reads,

$\alpha_{s}(\mu)=\frac{g^{2}(\mu)}{4 \pi}=\frac{6 \pi}{\left(33-2 N_{f}\right) \ln (a \mu)}$

$$
\times\left[1-\frac{3\left(153-19 N_{f}\right)}{\left(33-2 N_{f}\right)^{2}} \frac{\ln (2 \ln (a \mu))}{\ln (a \mu)}\right],
$$

where $a=1.91 /(2.91 \zeta)$, and $\zeta$ is a phenomenological parameter of the quasiparticle model, which characterizes the strength of the non-perturbative effect. The larger $\zeta$ brings the stronger interaction $\alpha_{s}(\mu)$.

When the full quark propagator is specified, using the contour integration method, the quark number density for each quark flavour reads,

$\rho_{f}(\mu)=\frac{N_{c}}{3 \pi^{2}}\left(\mu^{2}-m_{f}^{2}(\mu)\right)^{3 / 2} \theta\left(\mu-m_{f}(\mu)\right)$.

Since there is a step function on the right-hand side of Eq. (6), the quark number density vanishes when the quark chemical potential $\mu$ is less than a critical value $\mu_{c}$. This phenomenon is qualitatively consistent with the general conclusions in Ref. [22]. Here, the critical chemical potential depends on the current quark mass $m_{f 0}$ and the parameter $\zeta$. For the case of chiral limit, i.e., $m_{f 0}=0$, one can obtain $\mu_{c} \doteq 2.23 \zeta$.

Then, according to Refs. [23,24], the pressure of strange quark matter at zero temperature and finite chemical potential can be written as,

$P(\mu)=\left.P(\mu)\right|_{\mu=0}+\int_{0}^{\mu} \mathrm{d} \mu^{\prime} \rho\left(\mu^{\prime}\right)$,

where $\left.P(\mu)\right|_{\mu=0}$ is called the vacuum pressure which defines the pressure at $\mu=0$. And it can also be written as $\left.P(\mu)\right|_{\mu=0} \equiv-B(B>0)$, where $B$ is also a phenomenological parameter in our present model. Since the parameter $B$ is analogous to the bag constant in the MIT bag model, it is always a positive number, for the vacuum pressure must be negative, which preserves the confinement of quarks and gluons. It should also be noticed that Eq. (7) is a model independent expression, since the pressure $P(\mu)$ at zero temperature and finite chemical potential is determined by the quark number density $\rho(\mu)$ (up to a constant $\left.P(\mu)\right|_{\mu=0}$ ). Therefore, once the quark number density $\rho(\mu)$ matches the phenomena of QCD in low density region, the pressure surely satisfies the behavior of QCD at the same region.

In strange quark stars, there are $u, d$, and $s$ quarks in such complex system, and the baryon number density of this system is defined as,

$\rho_{B}(\mu) \equiv \frac{1}{3}\left(\rho_{u}\left(\mu_{u}\right)+\rho_{d}\left(\mu_{d}\right)+\rho_{s}\left(\mu_{s}\right)\right)$,

and the pressure of such system is given by,

$P(\mu)=-B+P_{u}\left(\mu_{u}\right)+P_{d}\left(\mu_{d}\right)+P_{s}\left(\mu_{s}\right)+P_{e}\left(\mu_{e}\right)$.

When considering the electroweak effects, one should take the constraints of chemical equilibrium and charge neutrality 
conditions, such like,

$$
\begin{aligned}
\mu_{d} & =\mu_{u}+\mu_{e}, \\
\mu_{s} & =\mu_{u}+\mu_{e}, \\
\frac{2}{3} \rho_{u} & =\frac{1}{3} \rho_{s}+\frac{1}{3} \rho_{d}+\rho_{e},
\end{aligned}
$$

where, the number density of electrons at zero temperature is given by [25],

$$
\rho_{e}\left(\mu_{e}\right)=\frac{\mu_{e}^{3}}{3 \pi^{2}} .
$$

Owing to the constraints given by Eqs. (10), (11) and (12), there is just one independent chemical potential left. In this work, $\mu_{u}$ is treated as the variable, and other chemical potentials, such as $\mu_{d}, \mu_{s}$, and $\mu_{e}$, are expressed as functions of $\mu_{u}$.

Finally, the energy density of this model is given by,

$$
\begin{aligned}
\epsilon & =-P+\mu \cdot \frac{\partial P}{\partial \mu} \\
& =-P+\sum_{f=u, d, s, e} \mu_{f} \rho_{f}\left(\mu_{f}\right) .
\end{aligned}
$$

From Eq. (14), we can see that the EOS described by this quasiparticle model depends on the masses of current quarks, the parameter $\zeta$, and the so-called vacuum pressure $B$.

\section{The properties of strange quark stars}

When the EOS of strange quark matter is constructed, it is now straightforward to explore the inner structures of strange quark stars. As we all know, the tidal Love number describes that how easily the substantial part of a star is deformed by an external tidal field, as discussed in [26-28], which is associated with the dominant contribution to the stellar deformation, while the tidal deformability describes the ratio of each star's induced mass quadrupole moment to the tidal field of its companion $[26,29,30]$. The perturbation scheme developed in $[27,31,32]$ is used to calculate the $l=2$ dimensionless tidal Love number $k_{2}$ and the associated tidal deformability $\Lambda$. And they are related by (in the conventions: $G=c=1$ ),

$\Lambda=\frac{2}{3} k_{2}\left(\frac{R}{M}\right)^{5}$.

In order to compute the tidal deformabilities for strange quark stars using our EOS, the Tolman-OppenheimerVolkoff (TOV) equations [33,34]

$$
\begin{aligned}
\frac{\mathrm{d} P(r)}{\mathrm{d} r} & =-\frac{(\epsilon+P)\left(M+4 \pi r^{3} P\right)}{r(r-2 M)}, \\
\frac{\mathrm{d} M(r)}{\mathrm{d} r} & =4 \pi r^{2} \epsilon,
\end{aligned}
$$

should be solved simultaneously with

$$
\begin{aligned}
& \frac{\mathrm{d} H(r)}{\mathrm{d} r}=\beta, \\
& \frac{\mathrm{d} \beta(r)}{\mathrm{d} r}=2\left(1-2 \frac{M}{r}\right)^{-1} \\
& \quad H\{-2 \pi[5 \epsilon+9 P+f(\epsilon+P)] \\
& \left.+\frac{3}{r^{2}}+2\left(1-2 \frac{M}{r}\right)^{-1}\left(\frac{M}{r^{2}}+4 \pi r P\right)^{2}\right\} \\
& +\frac{2 \beta}{r}\left(1-2 \frac{M}{r}\right)^{-1}\left\{-1+\frac{M}{r}+2 \pi r^{2}(\epsilon-P)\right\},
\end{aligned}
$$

where, $f$ is written by

$$
f=\frac{\mathrm{d} \epsilon}{\mathrm{d} P}
$$

for slow changes in matter configurations. Equations (18) and (19) are integrated outward starting just outside the center of strange quark stars via the expansions: $H(r)=a_{0} r^{2}$, and $\beta(r)=2 a_{0} r$ as $r \rightarrow 0$, where $a_{0}$ is a constant which determines how much the star is deformed by an external tidal field and can be chosen arbitrarily since it is cancelled in the expression of the Love number $k_{2}$. Here, in our calculations, we choose $a_{0}=1$ for simplicity.

For the internal solutions, the $l=2$ tidal Love number $k_{2}$ reads

$$
\begin{aligned}
k_{2}= & \frac{8 C^{5}}{5}(1-2 C)^{2}\left[2+2 C\left(y_{R}-1\right)-y_{R}\right] \\
& \times\left\{2 C\left[6-3 y_{R}+3 C\left(5 y_{R}-8\right)\right]\right. \\
& +4 C^{3}\left[13-11 y_{R}+C\left(3 y_{R}-2\right)+2 C^{2}\left(1+y_{R}\right)\right] \\
& \left.+3(1-2 C)^{2}\left[2-y_{R}+2 C\left(y_{R}-1\right)\right] \ln (1-2 C)\right\}^{-1},
\end{aligned}
$$

where $C=M / R$ is the compactness of the star, and $y_{R}$ is given by $y_{R}=y(R)=[r \beta(r) / H(r)]_{r=R}$.

As discussed in Refs. [35,36], if there is a first-order quark-hadron phase transition in the EOS, there is a jump of $\Delta \epsilon$ in the energy density at pressure $P_{t r}$ where this transition happens. This delta-function behavior should be considered in Eq. (20) as

$f=\left.\frac{\mathrm{d} \epsilon}{\mathrm{d} P}\right|_{P \neq P_{t r}}+\delta\left(P-P_{t r}\right) \Delta \epsilon$.

This leads to an additional term for the solution of $y(r)$ across $r_{t r}$, which is given by

$y\left(r_{t r}^{+}\right)-y\left(r_{t r}^{-}\right)=-\frac{4 \pi r_{t r}^{3} \Delta \epsilon}{M\left(r_{t r}\right)+4 \pi r_{t r}^{3} P\left(r_{t r}\right)}$, 
where $r_{t r}$ is the position where this first-order phase transition happens in the star, $\Delta \epsilon$ is given by $\Delta \epsilon=\epsilon\left(r_{t r}^{-}\right)-\epsilon\left(r_{t r}^{+}\right)$, and $r_{t r}^{ \pm}=r_{t r} \pm \delta r$ where $\delta r$ is an infinitesimal distance around $r_{t r}$.

For strange quark stars, since there is a finite energy density discontinuity in the vicinity of the surface $R$, one should also add this extra term to $y(R)$ and get

$y_{R}=\frac{R \beta(R)}{H(R)}-\frac{4 \pi R^{3} \Delta \epsilon}{M+4 \pi R^{3} P(R)}$,

where $\Delta \epsilon=\epsilon\left(R^{-}\right)$is the energy density just inside the surface, and the pressure at the surface is given by $P(R)=0$.

For the parameters, we choose $\zeta$ changing from $0.05 \mathrm{GeV}$ to $0.095 \mathrm{GeV}$, since in this region the running coupling constant $\alpha_{s}(\mu)$ varies consistent with the experimental data [37]. The vacuum pressure $B$ varies from $(0.098 \mathrm{GeV})^{4}$ to $(0.148$ $\mathrm{GeV})^{4}$, which ensures that the surface energy density is always larger than $2.80 \times 10^{14} \mathrm{~g} / \mathrm{cm}^{3}$, and the energy per baryon number at zero pressure is smaller than that for the ordinary nuclear matter. For the mass of $u$ or $d$ quark is very small, we set $m_{u 0}=m_{d 0}=0 \mathrm{MeV}$. The masses of s quark are $0 \mathrm{MeV}, 30 \mathrm{MeV}, 60 \mathrm{MeV}, 90 \mathrm{MeV}$ and $120 \mathrm{MeV}$. Every set of parameters is made by different mass of s quark with the same values for $\zeta$ and $B$, and of course the same mass of $\mathrm{u}$ or $\mathrm{d}$ quark. And, the effect of the strange quark mass on the observables of strange quark stars is analysed in the followings.

All the results we present in this paper are obtained from the astrophysically compatible EOSs that are selected by two astrophysical constraints: one is the mass constraint of $M_{\mathrm{TOV}}>2.0 M_{\odot}$ that comes from the observables, PSR J1614-2230 $\left(M=1.928 \pm 0.017 M_{\odot}\right.$ [2] $)$ and PSR $\mathrm{J} 0348+0432\left(M=2.01 \pm 0.04 M_{\odot}[1]\right)$, where $M_{\mathrm{TOV}}$ is the maximum mass for each EOS while solving the TOV equations, and the other is the constraint on the tidal deformability of $\Lambda_{1.4}<800$ from GW170817 [3]. In Figs. 1, 2, 3, and 8 , the quantities shown in blue-solid, magenta-solid, and yellow-solid lines represent the results calculated with $m_{s 0}=$ $0 \mathrm{MeV}, 60 \mathrm{MeV}$, and $120 \mathrm{MeV}$, respectively. And in Figs. 4, 5,6 , and 7 , the quantities shown in blue-solid, magenta-solid, and yellow-solid lines stand for their upper bounds calculated with $m_{s 0}=0 \mathrm{MeV}, 60 \mathrm{MeV}$, and $120 \mathrm{MeV}$, respectively, while the quantities shown in blue-dashed, magenta-dashed, and yellow-dashed lines stand for their lower bounds calculated with $m_{s 0}=0 \mathrm{MeV}, 60 \mathrm{MeV}$, and $120 \mathrm{MeV}$, respectively.

In order to check that strange quark matter that described by the astrophysically compatible EOSs are more stable than the ordinary nuclear matter EOS, we extract the relation between energy per baryon number and baryon number density from the EOS described using the Compressible Liquid Drop Model (CLDM) of nuclei with the parameters calculated using the SLy effective nucleon-nucleon interac-

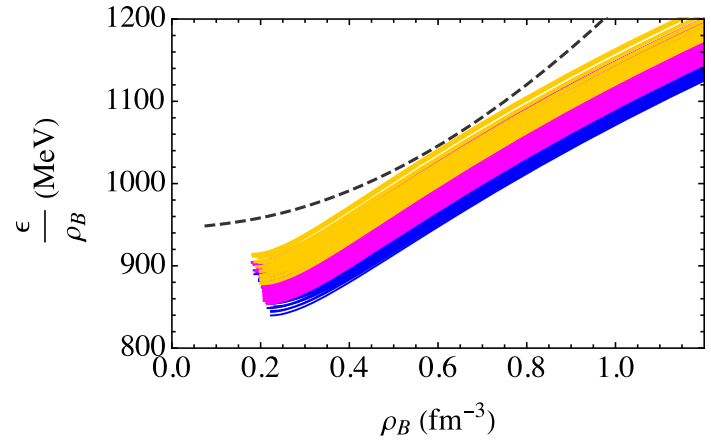

Fig. 1 The relation between energy per baryon and baryon number density. The black-dashed line represents this relation for the EOS of ordinary nuclear matter described by CLDM
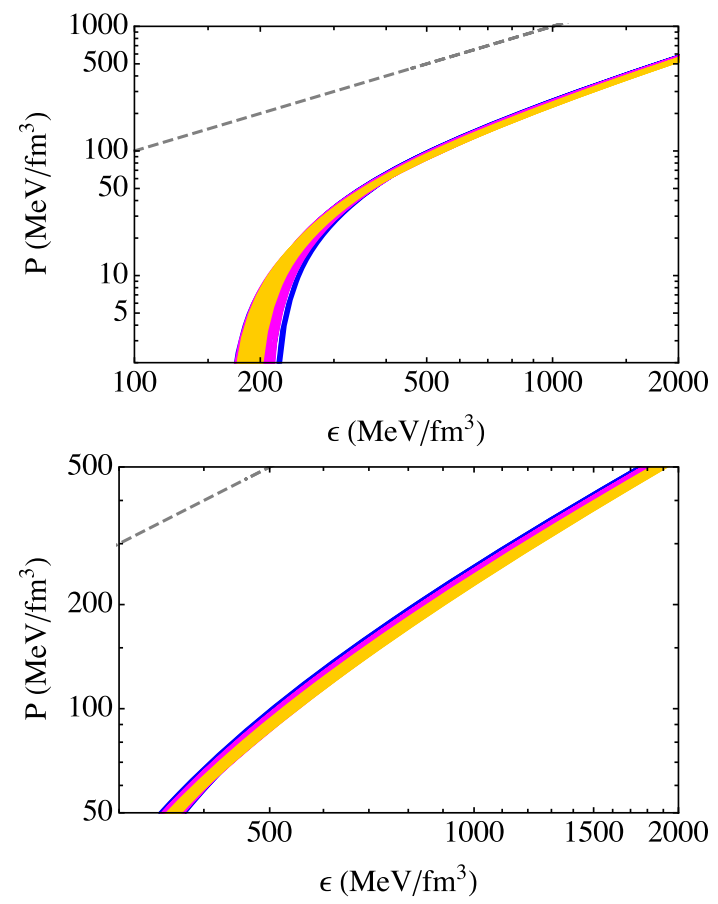

Fig. 2 Equations of state for strange quark stars selected by the constraints of $M_{\mathrm{TOV}}>2 M_{\odot}$ and $\Lambda_{1.4}<800$. The gray-dashed line stands for $P=\epsilon$

tions [38], and compare this relation with that extracted from the astrophysically compatible EOSs in this work. Figure 1 shows this comparison. And it is obviously that the energy per baryon number for the astrophysically compatible EOSs are smaller than that for the EOS of ordinary nuclear matter described by CLDM, which fulfills Witten's conjecture [39].

The equations of state for strange quark matter with different strange quark masses are shown in Fig. 2. And the gray-dashed line stands for $P=\epsilon$. From this picture, we can see that in the low density region the astrophysically compatible EOSs with large strange quark mass are stiffer, while in the high density region they are softer. 

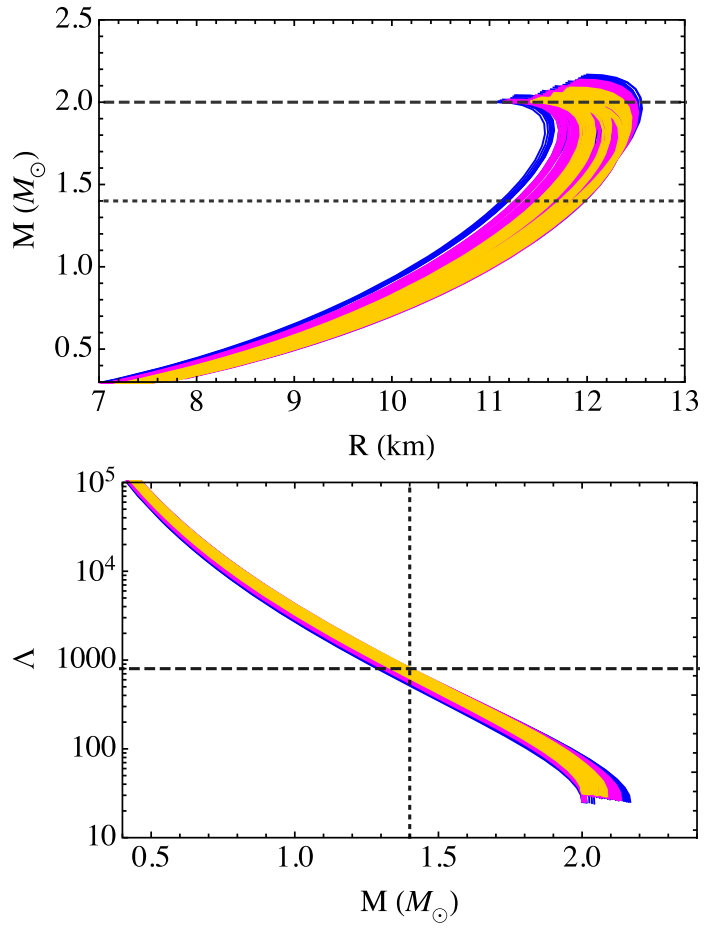

Fig. 3 Upper: mass-radius relations for strange quark stars. The blackdashed line stands for $M=2.0 M_{\odot}$, and the black-dotted line stands for $M=1.4 M_{\odot}$. Lower: $\Lambda$-mass relations for strange quark stars. The black-dashed horizontal line stands for $\Lambda=800$, and the black-dotted vertical line stands for $M=1.4 M_{\odot}$

The mass-radius relations of the astrophysically compatible EOSs are shown in the upper panel of Fig. 3. The blackdashed line stands for $M=2.0 M_{\odot}$, and the black-dotted line stands for $M=1.4 M_{\odot}$. And the $\Lambda$-mass relations of the astrophysically compatible EOSs are shown in the lower panel of Fig. 3. The black-dashed horizontal line stands for $\Lambda=800$, and the black-dotted vertical line stands for $M=1.4 M_{\odot}$.

Our results for the masses, radii and tidal deformabilities with different strange quark masses are listed in Table 1. The chemical potentials for $\mathrm{u}, \mathrm{d}$, and s quarks $\left(\mu_{u, d}, \mu_{s}\right)$ at the surface of strange quark stars are also listed, and at these chemical potentials the quasiparticle model is still valid. The constraint on tidal deformability of $\Lambda_{1.4}<800$ gives the

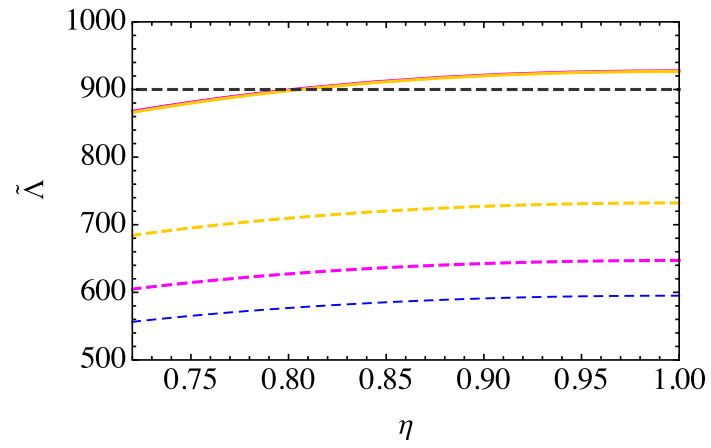

Fig. 4 Combined tidal deformability $\tilde{\Lambda}$ as a function of mass ratio $\eta$ for the case of GW170817. The black-dashed line stands for $\tilde{\Lambda}=900$

maximum mass and radius for strange quark stars. A larger strange quark mass gives a smaller mass and radius for a strange quark star, and, the variation of the maximum radius $R_{1.4}$ for $1.4 M_{\odot}$ strange quark stars is very small. The mass constraint of $M_{\mathrm{TOV}}>2.0 M_{\odot}$ gives the minimum radius, and a larger strange quark mass gives a larger minimum of radius for a $1.4 M_{\odot}$ strange quark star. The mass constraint also gives the minimum tidal deformability, and the minimum of tidal deformabilities for $1.4 M_{\odot}$ strange quark stars become larger for larger values of strange quark mass.

Now, we consider the tidal deformabilities of two compact stars in the binary inspiral. The chirp mass reads

$\mathcal{M}=\frac{\left(m_{1} m_{2}\right)^{3 / 5}}{\left(m_{1}+m_{2}\right)^{1 / 5}}$

where, $m_{1}$ and $m_{2}$ are the masses of the two compact stars. And the combined tidal deformability is given by

$\tilde{\Lambda}=\frac{16}{13} \frac{\left(m_{1}+12 m_{2}\right) m_{1}^{4} \Lambda_{1}+\left(m_{2}+12 m_{1}\right) m_{2}^{4} \Lambda_{2}}{\left(m_{1}+m_{2}\right)^{5}}$,

where, $\Lambda_{1}$ and $\Lambda_{2}$ are the tidal deformabilities of the two compact stars.

In the low-spin case of GW170817 [3,4], the chirp mass is $\mathcal{M}=1.186_{-0.001}^{+0.001} M_{\odot}$, the component masses are $m_{1} \in$ $[1.36,1.60] M_{\odot}, m_{2} \in[1.16,1.36] M_{\odot}$, and the mass ratio is $\eta=m_{2} / m_{1} \in[0.72,1.0]$.

the minimum radius $R_{\min }$, and the maximum radius $R_{1.4 \max }$, the minimum radius $R_{1.4 \mathrm{~min}}$ and the minimum tidal deformability $\Lambda_{1.4 \mathrm{~min}}$ for $1.4 M_{\odot}$ strange quark stars, with different strange quark masses $m_{s 0}$
Table 1 Properties of the strange quark stars, including the chemical potentials of $\mathrm{u}, \mathrm{d}$, and s quarks, $\mu_{u, d}$ and $\mu_{s}$, at the surface of strange quark stars, the maximum mass $M_{\max }$, the maximum radius $R_{\max }$ and

\begin{tabular}{lllllllll}
\hline$m_{s 0}(\mathrm{MeV})$ & $\mu_{u, d}(\mathrm{GeV})$ & $\mu_{s}(\mathrm{GeV})$ & $M_{\max }\left(M_{\odot}\right)$ & $R_{\max }(\mathrm{km})$ & $R_{\min }(\mathrm{km})$ & $R_{1.4 \max }(\mathrm{km})$ & $R_{1.4 \min }(\mathrm{km})$ & $\Lambda_{1.4 \min }$ \\
\hline 0 & $0.279-0.299$ & $0.279-0.299$ & 2.168 & 12.001 & 11.066 & 11.977 & 11.118 \\
30 & $0.284-0.305$ & $0.290-0.311$ & 2.156 & 11.982 & 11.100 & 11.973 & 11.180 \\
60 & $0.283-0.303$ & $0.296-0.317$ & 2.134 & 11.929 & 11.149 & 11.970 & 11.268 \\
90 & $0.281-0.300$ & $0.302-0.322$ & 2.115 & 11.899 & 11.387 & 11.963 & 11.385 & 596.1 \\
120 & $0.281-0.296$ & $0.313-0.328$ & 2.086 & 11.828 & 11.504 & 11.957 & 11.494 & 632.6 \\
\hline
\end{tabular}


Table 2 Combined tidal deformabilities of strange quark stars for the case of GW170817: the ratio $\left.\tilde{\Lambda}\right|_{\eta=0.72} /\left.\tilde{\Lambda}\right|_{\eta=1.0}$, the upper bound of combined tidal deformability $\tilde{\Lambda}_{U B}$, the lower bound of combined tidal deformability $\tilde{\Lambda}_{L B}$, with different strange quark masses $m_{s 0}$

\begin{tabular}{llll}
\hline$m_{s 0}(\mathrm{MeV})$ & $\left.\tilde{\Lambda}\right|_{\eta=0.72} /\left.\tilde{\Lambda}\right|_{\eta=1.0}$ & $\tilde{\Lambda}_{U B}$ & $\tilde{\Lambda}_{L B}$ \\
\hline 0 & $93.38-93.58 \%$ & $868.0-927.5$ & $556.4-595.1$ \\
30 & $93.39-93.56 \%$ & $867.0-926.7$ & $576.0-616.1$ \\
60 & $93.39-93.53 \%$ & $867.5-927.5$ & $604.9-647.2$ \\
90 & $93.39-93.51 \%$ & $866.9-927.0$ & $645.4-690.6$ \\
120 & $93.39-93.47 \%$ & $866.1-926.6$ & $684.2-732.2$ \\
\hline
\end{tabular}

Shown in Fig. 4 is the combined tidal deformability $\tilde{\Lambda}$ as a function of the mass ratio $\eta$, which are calculated by the astrophysically compatible EOSs for strange quark stars. The upper bounds for $\tilde{\Lambda}$ with different strange quark mass almost coincide. The black-dashed line stands for $\tilde{\Lambda}=900$.

The results for the combined tidal deformability with different strange quark masses are listed in Table 2 . The ratio of $\left.\tilde{\Lambda}\right|_{\eta=0.72}$ to $\left.\tilde{\Lambda}\right|_{\eta=1.0}$ is stable when varying the strange quark mass. The upper bound of $\tilde{\Lambda}_{U B}$ is also stable, while the lower bound of $\tilde{\Lambda}_{L B}$ becomes larger with increasing strange quark mass.

The two tidal parameters $\Lambda_{1}$ and $\Lambda_{2}$ are also shown in Fig. 5 with the $90 \%$ probability contours for the tidal deformabilities of the two stars measured by LIGO and Virgo. The purple-solid line stands for the $90 \%$ probability contours given in Fig. 5 of Ref. [3], and the brown-solid line represents the $90 \%$ probability contours given in Fig. 10 of Ref. [4]. Both of them are for the waveform models of TaylorF2. The brown lines are calculated using a lower starting frequency of $23 \mathrm{~Hz}$ instead of $30 \mathrm{~Hz}$, resulting in the upper bounds of $\Lambda_{1}$ and $\Lambda_{2}$ are about $20 \%$ smaller than the purple lines. The upper limits for $\left(\Lambda_{1}, \Lambda_{2}\right)$ with different strange quark mass almost coincide, while the lower limits for $\left(\Lambda_{1}, \Lambda_{2}\right)$ with larger strange quark mass stays closer to the $90 \%$ probability contour.

In the low-spin case of GW190425 [40], the chirp mass is $\mathcal{M}=1.44_{-0.02}^{+0.02} M_{\odot}$, the component masses are $m_{1} \in$ $[1.60,1.87] M_{\odot}, m_{2} \in[1.46,1.69] M_{\odot}$, and the mass ratio is $\eta=m_{2} / m_{1} \in[0.8,1.0]$.

The combined tidal deformabilities $\tilde{\Lambda}$ s for GW190425 as a function of mass ratio $\eta$ are shown in Fig. 6, which are calculated by the astrophysically compatible EOSs for strange quark stars. The black-dashed line stands for $\tilde{\Lambda}=$ 310.1, which is the maximum value for $\eta=0.8$ and $m_{s 0}=0$ [12].

The results for the combined tidal deformability with different strange quark masses are listed in Table 3. The ratio of $\left.\tilde{\Lambda}\right|_{\eta=0.8}$ to $\left.\tilde{\Lambda}\right|_{\eta=1.0}$ is also stable and larger than that in the case of GW170817 when varying the strange quark mass. Again, a larger strange quark mass gives a larger lower bound

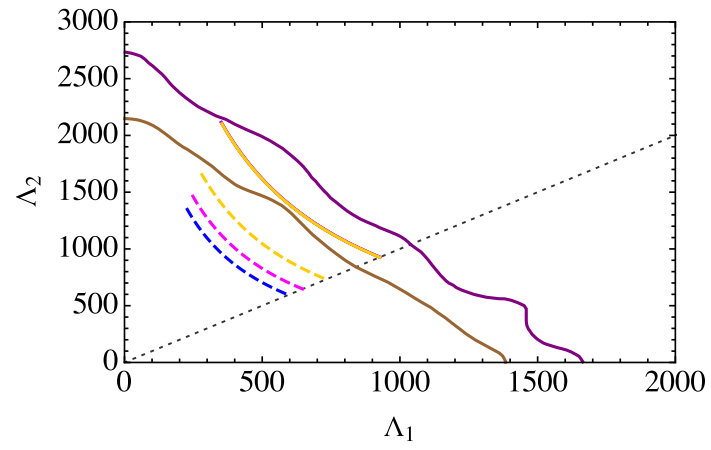

Fig. $5\left(\Lambda_{1}, \Lambda_{2}\right)$ relation obtained for the case of GW170817. The purple-solid line stands for the $90 \%$ probability contours given in Fig. 5 of Ref. [3], and the brown-solid line represents the $90 \%$ probability contours given in Fig. 10 of Ref. [4]. The black-dotted line stands for $\Lambda_{1}=\Lambda 2$

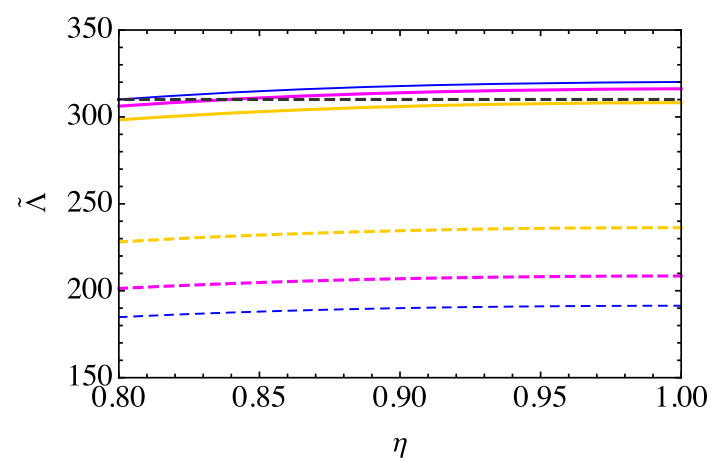

Fig. 6 Combined tidal deformability $\tilde{\Lambda}$ as a function of mass ratio $\eta$, calculated by the astrophysically compatible EOSs for the case of GW190425. The black-dashed line stands for $\tilde{\Lambda}=310.1$

Table 3 Combined tidal deformabilities of strange quark stars for the case of GW190425: the ratio $\left.\tilde{\Lambda}\right|_{\eta=0.8} /\left.\tilde{\Lambda}\right|_{\eta=1.0}$, the upper bound of combined tidal deformability $\tilde{\Lambda}_{U B}$, the lower bound of combined tidal deformability $\tilde{\Lambda}_{L B}$, with different strange quark masses $m_{s 0}$

\begin{tabular}{llll}
\hline$m_{s 0}(\mathrm{MeV})$ & $\left.\tilde{\Lambda}\right|_{\eta=0.8} /\left.\tilde{\Lambda}\right|_{\eta=1.0}$ & $\tilde{\Lambda}_{U B}$ & $\tilde{\Lambda}_{L B}$ \\
\hline 0 & $96.54-96.95 \%$ & $310.1-320.1$ & $184.8-191.4$ \\
30 & $96.54-96.93 \%$ & $308.6-318.6$ & $191.5-198.3$ \\
60 & $96.54-96.87 \%$ & $306.2-316.2$ & $201.3-208.5$ \\
90 & $96.52-96.84 \%$ & $304.9-314.9$ & $215.2-215.4$ \\
120 & $96.49-96.78 \%$ & $298.3-308.2$ & $228.1-236.3$ \\
\hline
\end{tabular}

of $\tilde{\Lambda}_{L B}$ and a little bit smaller upper bound of $\tilde{\Lambda}_{U B}$, i.e., a narrower range of $\tilde{\Lambda}$.

The two tidal parameters $\Lambda_{1}$ and $\Lambda_{2}$ are also shown in Fig. 7.

Finally, the relation between $\tilde{\Lambda}$ and $\mathcal{M}$ are also presented in Fig. 8. The upper panel shows the $\tilde{\Lambda}-\mathcal{M}$ relations for the mass ratio $\eta=0.7$, while the lower panel shows the $\tilde{\Lambda}$ $\mathcal{M}$ relations for the mass ratio $\eta=1.0$. The black-dashed horizontal line stands for $\tilde{\Lambda}=900$, while the two blackdotted vertical lines stand for the chirp mass in the case of 


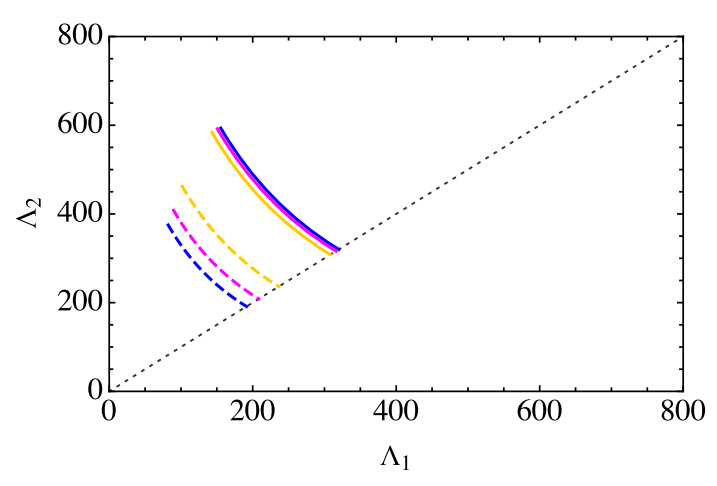

Fig. $7\left(\Lambda_{1}, \Lambda_{2}\right)$ relation obtained for the case of GW190425. The black-dotted line stands for $\Lambda_{1}=\Lambda 2$
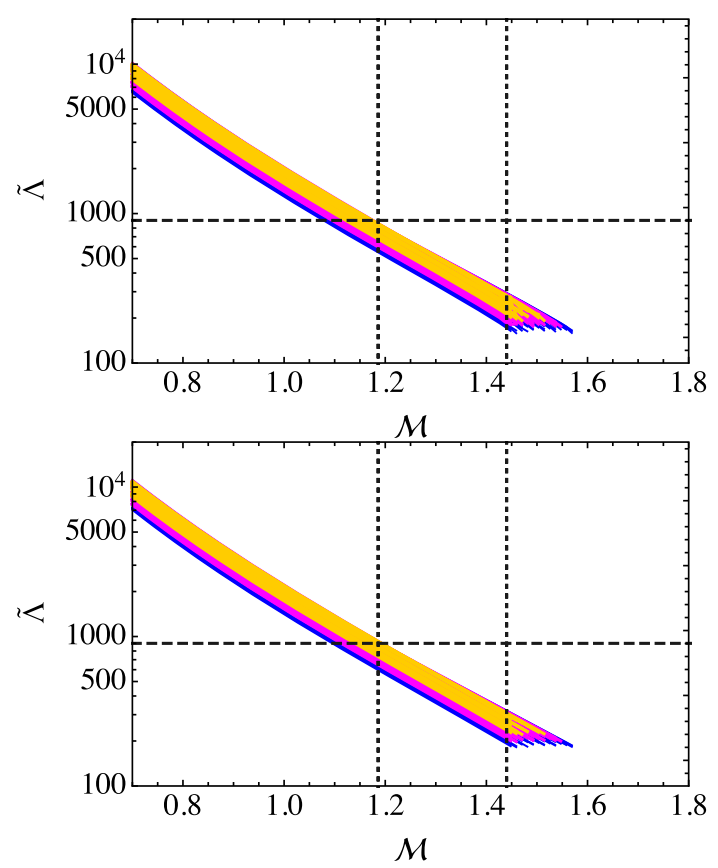

Fig. 8 Combined tidal deformability $\tilde{\Lambda}$ as a function of chirp mass, calculated by the astrophysically compatible EOSs for the case of $\eta=0.7$ (Upper) and $\eta=1.0$ (Lower), respectively. The black-dashed horizontal line stands for $\tilde{\Lambda}=900$, while the two black-dotted vertical lines stand for $\mathcal{M}=1.186$ and $\mathcal{M}=1.44$, respectively

GW170817 and GW190425, i.e., $\mathcal{M}=1.186$ and $\mathcal{M}=$ 1.44, respectively. The same as shown in Tables 2 and $3, \tilde{\Lambda} \mathrm{s}$ for $\eta=1.0$ are larger than that for $\eta=0.7$ with different chirp masses, and the lower bound of $\tilde{\Lambda}$ for a lager strange quark mass is larger than that for a smaller strange quark mass.

\section{Summary}

The effects of strange quark mass on masses, tidal deformabilities and radii of strange quark stars are studied via the quasiparticle model that includes the non-perturbative fea- tures of QCD in the low-density region. The constraints of $M_{\mathrm{TOV}}>2.0 M_{\odot}$ and $\Lambda_{1.4}<800$ are used to choose the EOSs with different strange quark masses. Our results show that, in the low density region, the astrophysically compatible EOSs with large strange quark mass selected by these two astrophysical constraints are stiffer, while in the high density region they are softer.

Table 1 lists the results for the masses, radii and tidal deformabilities with different strange quark masses. These data shows that a larger strange quark mass gives a smaller mass and radius for a strange quark star, and a larger minimum of radii and tidal deformabilities for $1.4 M_{\odot}$ strange quark stars, and the variation of the maximum radius $R_{1.4}$ for $1.4 M_{\odot}$ strange quark stars is very small.

In the case of GW170817, results in Table 2 show that, a larger strange quark mass gives a larger lower bound of $\tilde{\Lambda}$, while the upper bound of $\tilde{\Lambda}$ is stable.

In the case of GW190425, results in Table 3 show that, a larger strange quark mass gives a larger lower bound of $\tilde{\Lambda}$ and a little bit smaller upper bound of $\tilde{\Lambda}$, i.e., a narrower range of $\tilde{\Lambda}$.

The relation between $\tilde{\Lambda}$ and $\mathcal{M}$ is also calculated. The same as shown in Tables 2 and $3, \tilde{\Lambda}$ s for $\eta=1.0$ are larger than that for $\eta=0.7$ with different chirp masses, and the lower bound of $\tilde{\Lambda}$ for lager strange quark mass is larger than that for smaller strange quark mass.

Acknowledgements This work is supported by the National Natural Science Foundation of China (under Grant nos. 11805097, 11775118, and 11535005), the Jiangsu Provincial Natural Science Foundation of China (under Grant no. BK20180323), the China Postdoctoral Science Foundation (under Grant no. 2020M681655), and the Jiangsu Planned Projects for Postdoctoral Research Funds (under Grants no. 2020Z271).

Data Availability Statement This manuscript has no associated data or the data will not be deposited. [Authors' comment: The results in the current article are based solely on the authors' computations, and there is no further associated data.]

Open Access This article is licensed under a Creative Commons Attribution 4.0 International License, which permits use, sharing, adaptation, distribution and reproduction in any medium or format, as long as you give appropriate credit to the original author(s) and the source, provide a link to the Creative Commons licence, and indicate if changes were made. The images or other third party material in this article are included in the article's Creative Commons licence, unless indicated otherwise in a credit line to the material. If material is not included in the article's Creative Commons licence and your intended use is not permitted by statutory regulation or exceeds the permitted use, you will need to obtain permission directly from the copyright holder. To view a copy of this licence, visit http://creativecomm ons.org/licenses/by/4.0/.

Funded by $\mathrm{SCOAP}^{3}$. 


\section{References}

1. J. Antoniadis, P.C.C. Freire, N. Wex, T.M. Tauris, R.S. Lynch, M.H. van Kerkwijk, M. Kramer, C. Bassa, V.S. Dhillon, T. Driebe, J.W.T. Hessels, V.M. Kaspi, V.I. Kondratiev, N. Langer, T.R. Marsh, M.A. McLaughlin, T.T. Pennucci, S.M. Ransom, I.H. Stairs, J. van Leeuwen, J.P.W. Verbiest, D.G. Whelan, Science (2013). https:// doi.org/10.1126/science.1233232

2. E. Fonseca, T.T. Pennucci, J.A. Ellis, I.H. Stairs, D.J. Nice, S.M. Nsom, P.B. Demorest, Z. Arzoumanian, K. Crowter, T. Dolch, R.D. Ferdman, M. Gonzalez, G. Jones, M.L. Jones, M.T. Lam, L. Levin, M.A. McLaughlin, K. Ovall, J.K. Swiggum, W. Zhu, Astrophys. J. 832, 167 (2016)

3. B.P. Abbott, R. Abbott, T.D. Abbott, F. Acernese, K. Ackley, C. Adams, T. Adams, P. Addesso et al., LIGO Scientific Collaboration and Virgo Collaboration. Phys. Rev. Lett. 119, 161101 (2017)

4. B.P. Abbott, R. Abbott, T.D. Abbott, F. Acernese, K. Ackley, C. Adams, T. Adams, P. Addesso et al., LIGO Scientific Collaboration and Virgo Collaboration. Phys. Rev. X 9, 011001 (2019)

5. G. Baym, T. Hatsuda, T. Kojo, P.D. Powell, Y. Song, T. Takatsuka, Rep. Progr. Phys. 81, 056902 (2018)

6. E.-P. Zhou, X. Zhou, A. Li, Phys. Rev. D 97, 083015 (2018)

7. S. Han, M.A.A. Mamun, S. Lalit, C. Constantinou, M. Prakash, Phys. Rev. D 100, 103022 (2019)

8. K. Masuda, T. Hatsuda, T. Takatsuka, Progr. Theor. Exp. Phys. (2013). https://doi.org/10.1093/ptep/ptt045. https://arxiv.org/ abs/https://academic.oup.com/ptep/article-pdf/2013/7/073D01/ 19300294/ptt045.pdf

9. A. Peshier, B. Kämpfer, G. Soff, Phys. Rev. C 61, 045203 (2000)

10. B.-L. Li, Z.-F. Cui, Z.-H. Yu, Y. Yan, S. An, H.-S. Zong, Phys. Rev. D 99, 043001 (2019)

11. Y. Yan, J. Cao, X.-L. Luo, W.-M. Sun, H. Zong, Phys. Rev. D 86, 114028 (2012)

12. B.-L. Li, Y. Yan, J.-L. Ping, Phys. Rev. D 104, 043002 (2021)

13. A. Peshier, B. Kampfer, O. Pavlenko, G. Soff, Phys. Lett. B 337, 235 (1994)

14. M.I. Gorenstein, S.N. Yang, Phys. Rev. D 52, 5206 (1995)

15. V.M. Bannur, Phys. Rev. C 78, 045206 (2008)

16. V.M. Bannur, Phys. Rev. C 75, 044905 (2007)

17. H.-S. Zong, W.-M. Sun, Int. J. Mod. Phys. A 23, 3591 (2008a)

18. A.-M. Zhao, J. Cao, L.-J. Luo, W.-M. Sun, H.-S. Zong, Mod. Phys. Lett. A 25, 47 (2010)

19. W.E. Caswell, Phys. Rev. Lett. 33, 244 (1974)

20. R.A. Schneider, (2003) arXiv:hep-ph/0303104

21. H.-H. Ma, W.-L. Qian, Braz. J. Phys. 48, 160 (2018)
22. M.A. Halasz, A.D. Jackson, R.E. Shrock, M.A. Stephanov, J.J.M. Verbaarschot, Phys. Rev. D 58, 096007 (1998)

23. M. He, W.M. Sun, H.T. Feng, H.S. Zong, J. Phys. G Nucl. Part. 34, 2655 (2007)

24. H.-S. Zong, W.-M. Sun, Phys. Rev. D 78, 054001 (2008b)

25. J.I. Kapusta, C. Gale, Finite-Temperature Field Theory: Principles and Applications. Cambridge Monographs on Mathematical Physics, 2nd edn. (Cambridge University Press, Cambridge, 2006)

26. T. Damour, A. Nagar, Phys. Rev. D 80, 084035 (2009)

27. T. Hinderer, Astrophys. J. 677, 1216 (2008)

28. K. Yagi, N. Yunes, Science 341, 365 (2013)

29. T. Damour, M. Soffel, C. Xu, Phys. Rev. D 45, 1017 (1992)

30. T. Binnington, E. Poisson, Phys. Rev. D 80, 084018 (2009)

31. E.E. Flanagan, T. Hinderer, Phys. Rev. D 77, 021502 (2008)

32. T. Hinderer, B.D. Lackey, R.N. Lang, J.S. Read, Phys. Rev. D 81, 123016 (2010)

33. R.C. Tolman, Phys. Rev. 55, 364 (1939)

34. J.R. Oppenheimer, G.M. Volkoff, Phys. Rev. 55, 374 (1939)

35. S. Postnikov, M. Prakash, J.M. Lattimer, Phys. Rev. D 82, 024016 (2010)

36. J. Takátsy, P. Kovács, Phys. Rev. D 102, 028501 (2020)

37. S. Bethke, Prog. Part. Nucl. Phys. 58, 351 (2007)

38. F. Douchin, P. Haensel, A\&A 380, 151 (2001)

39. E. Witten, Phys. Rev. D 30, 272 (1984)

40. B.P. Abbott, R. Abbott, T.D. Abbott, F. Acernese, K. Ackley, C. Adams, T. Adams, P. Addesso et al., LIGO Scientific Collaboration and Virgo Collaboration. Astrophys. J. 892, L3 (2020) 